\title{
Variation in practice for preoperative antibiotic prophylaxis: a survey from an academic tertiary referral center in the United States
}

Nikhil Ailaney ${ }^{1 *} \mathbb{D}$, Elizabeth Zielinski², Michelle Doll³, Gonzalo M. Bearman³, Stephen L. Kates² and Gregory J. Golladay ${ }^{2}$

\begin{abstract}
Background: Antibiotic surgical prophylaxis is a core strategy for prevention of surgical site infections (SSI). Despite best practice guidelines and known efficacy of antibiotic prophylaxis in decreasing SSI risk, there is often wide variation in its use. This study was designed to determine the individual perspectives of perioperative providers at an academic tertiary referral center regarding their knowledge of preoperative antibiotic choice, dosing, and timing.

Methods: A prospective survey was conducted amongst surgical and anesthesia team members involved in preoperative antibiotic decision making. The survey addressed ten key principles relating to preoperative antibiotic use, including antibiotic choice, timing and rate of infusion, and dosing. The survey was distributed among orthopaedic surgeons, residents, and anesthesia providers at their respective monthly service line meetings between August 2017 to June 2019. The data was stored and analyzed in a Microsoft Excel worksheet.

Results: A total of 73 providers completed the survey. Twenty-two (30\%) of the providers agreed and 47 (64\%) disagreed that both vancomycin and cefazolin are equally effective for antibiotic prophylaxis. As for antibiotic choice in patients with penicillin allergies, 37 (51\%) agreed with vancomycin, 21 (29\%) agreed with clindamycin, and 15 (21\%) disagreed with both alternatives. When providers were surveyed regarding the appropriateness of standard versus weight adjusted dosing, 67 (92\%) agreed that vancomycin should be weight adjusted and 63 (86\%) agreed that cefazolin should be weight adjusted.

Conclusions: There is no clear consensus amongst providers for which antibiotic to administer for antibiotic prophylaxis despite existing guidelines. Discrepancy also exists between orthopaedic surgery and anesthesia providers in regards to appropriate antibiotic choice for patients with reported penicillin allergies. Institutions should implement evidence-based protocols for preoperative antibiotic prophylaxis and continue to prospectively monitor compliance in order to identify any inconsistencies that could result in inappropriate antibiotic prophylaxis for patients.
\end{abstract}

Keywords: Antibiotic prophylaxis, Surgical site infection, Orthopaedic surgery, Quality improvement

\footnotetext{
${ }^{*}$ Correspondence: nikhil_ailaney@urmc.rochester.edu

${ }^{1}$ Department of Orthopaedic Surgery, Virginia Commonwealth University

School of Medicine, 1200 Broad Street, 9th Floor, VA 23298 Richmond, USA

Full list of author information is available at the end of the article
}

\section{Background}

Surgical site infections (SSI) continue to be one of the most common complications after orthopaedic surgery $[1,2]$. Patients who develop SSI are at an increased risk of morbidity and mortality, often have longer hospital original author(s) and the source, provide a link to the Creative Commons licence, and indicate if changes were made. The images or other third party material in this article are included in the article's Creative Commons licence, unless indicated otherwise in a credit line to the material. If material is not included in the article's Creative Commons licence and your intended use is not permitted by statutory regulation or exceeds the permitted use, you will need to obtain permission directly from the copyright holder. To view a copy of this licence, visit http://creativecommons.org/licenses/by/4.0/. The Creative Commons Public Domain Dedication waiver (http://creativeco mmons.org/publicdomain/zero/1.0/) applies to the data made available in this article, unless otherwise stated in a credit line to the data. 
length of stays, and also have greater health care associated costs [3,4]. One of the most important strategies to reduce the risk of SSI is antibiotic prophylaxis, with a goal of decreasing the overall burden of microorganisms at the operative site [5]. Since the most common pathogen associated with SSI in orthopaedic procedures is Methicillin-sensitive Staphylococcus aureus (MSSA), antibiotics with excellent gram-positive coverage, such as first or third generation cephalosporins are often preferred. However, Methicillin-resistant Staphylococcus aureus (MRSA), Coagulase-negative Staphylococci (CoNS), and gram-negative bacilli are also important pathogens to consider. In addition, patient allergies, the side effect profile, and the cost associated with the antibiotic must also be considered.

The efficacy of antibiotic prophylaxis within the field of orthopaedic surgery is well documented. In total knee and total hip arthroplasty, a study reported an $81 \%$ decrease in risk of SSI with the use of antibiotic prophylaxis compared to without [6]. Similarly, in hip fracture surgery, a study reported almost $50 \%$ reduction in the rate of SSI with the use of antibiotic prophylaxis compared to without [2]. However, despite best practice guidelines and the known efficacy of antibiotic prophylaxis in reducing the risk of SSI, there is evidence of wide variation in antibiotic prophylaxis practices [7-9]. A study involving 2,965 hospitals, including 34,133 patients, determined that only $56 \%$ of patients received antibiotic prophylaxis within $60 \mathrm{~min}$ of the incision and another $20 \%$ of the patients received antibiotics between one and two hours before incision [7]. In addition, almost $10 \%$ of the patients received their first dose of antibiotics greater than four hours after the time of incision [7]. The authors also analyzed the time which antibiotics were discontinued and determined that antibiotics were discontinued within $24 \mathrm{~h}$ in only $41 \%$ of the patients studied [7].

Considering the effectiveness of antibiotic prophylaxis for decreasing the risk of SSI but the potential for great variability in its use despite best practice guidelines, we performed a qualitative study to assess the antibiotic prophylaxis perspectives of the orthopaedic surgery and anesthesiology teams at Virginia Commonwealth University (VCU) Health regarding preoperative antibiotic choice, dosing, and timing.

\section{Methods}

This study was conducted at an 850-bed tertiary care hospital with institutional pre-operative prophylaxis guidelines in place that prefer cefazolin with vancomycin as an alternative for penicillin allergy or an addition for MRSA-colonized patients. An Institution Review Board (IRB) approved survey (Fig. 1) was distributed amongst both orthopaedic surgery (nurse practitioners
(NP's), resident physicians, and attending physicians) and anesthesia (certified registered nurse anesthetists (CRNA's), resident physicians, and attending physicians) team members involved in preoperative antibiotic decision making from August 2017 to June 2019. The survey was distributed to all providers that met inclusion criteria during one monthly mandatory department meeting and anonymously collected at the end of the meeting. To meet inclusion criteria, providers that completed surveys had to be practicing resident physicians, advanced practice providers (NP's/CRNA's), or attending physicians within the orthopaedic surgery or anesthesiology departments at VCU Health. Medical students and ancillary surgical staff (surgical technologists, circulating nurses, and general perioperative nursing staff) were excluded from the study. Orthopaedic surgery residents and NP's were surveyed in August 2017, attending orthopaedic surgeons in October 2018, and anesthesia providers in June 2019. The survey addressed ten key practices relating to preoperative antibiotic use, including antibiotic choice for given clinical scenarios, timing and rate of antibiotic infusion, and antibiotic dosing. In addition, we collected opinions regarding barriers to timely antibiotic administration. After completion of the surveys by providers, the data was stored and analyzed in a Microsoft Excel worksheet.

\section{Results \\ Nurse practitioner and resident orthopaedic surgery providers}

A total of 2 orthopaedic NP's and 25 orthopaedic surgery residents were approached to complete the survey. Both NP's (100\%) and $22(88 \%)$ residents completed the survey. A total of 3 providers (13\%) agreed that vancomycin and cefazolin are equally effective for antibiotic prophylaxis whereas 19 (79\%) disagreed, and 2 (8\%) were unsure (Fig. 2). As for the antibiotic choice for patients with a penicillin allergy, 17 providers $(71 \%)$ agreed with vancomycin as the preferred alternative, 2 (8\%) preferred clindamycin, and 5 (21\%) disagreed with both practices. When providers were surveyed regarding the appropriateness of standard versus weight adjusted dosing, 22 (92\%) agreed that vancomycin should be dose adjusted by weight and 19 (79\%) agreed that cefazolin should be weight adjusted. Specific to vancomycin administration, the results indicated barriers to its effectiveness as a suitable method for prophylaxis. 22 providers (92\%) agreed that vancomycin infusion at the time of incision does not allow for adequate concentrations for appropriate antibiotic prophylaxis. In addition, 24 providers (100\%) recognized that vancomycin cannot be infused rapidly in order to maximize the proportion of dose infused prior to the time of incision. Furthermore, only 13 providers (54\%) 
Provider Questionnaire : Pre-operative Antibiotic Prophylaxis

For each statement or question, choose the ONE response that most closely fits you/your opinion.

1. Please identify your role:

a. Surgeon - attending d. Anesthesia - attending g. RN in OR

b. Surgeon -intern/resident/fellow e. Anesthesia-intern/resident/fellow h. RN in PSU

c. Surgical NP/PA f. Anesthesia NP i. Other

2. Vancomycin prophylaxis is as effective as Cefazolin prophylaxis in preventing surgical site infections. Strongly Agree Agree Disagree Strongly disagree Don't know

3. When a patient has a penicillin allergy, Vancomycin is preferred for prophylaxis Strongly Agree Agree Disagree Strongly disagree Don’t know

4. When a patient has a penicillin allergy, Clindamycin is preferred for prophylaxis Strongly Agree Agree Disagree Strongly disagree Don't know

5. When Vancomycin is used for prophylaxis, the infusion is usually completed by time of incision. Strongly Agree Agree Disagree Strongly disagree Don't know

6. Providers are usually aware of a patient's MRSA status when the patient arrives to the pre-op area Strongly Agree Agree Disagree Strongly disagree Don’t know

7. The standard dose of Vancomycin is $1000 \mathrm{mg}$, and adjustments for weight are generally not required. Strongly Agree Agree Disagree Strongly disagree Don't know

8. The standard dose of Cefazolin is $2 \mathrm{gm}$, and adjustments for weight are generally not required. Strongly Agree Agree Disagree Strongly disagree Don't know

9. We often discover a need to switch from Cefazolin to Vancomycin prophylaxis at the last minute. Strongly Agree Agree Disagree Strongly disagree Don't know

10. Vancomycin infusing at the time of incision produces adequate levels for optimal SSI prevention. Strongly Agree Agree Disagree Strongly disagree Don't know

11. Vancomycin can be infused rapidly in order to maximize the proportion of dose infused prior to the incision. Strongly Agree Agree Disagree Strongly disagree Don't know

12. What are the current barriers to administering Vancomycin infusions at least 60 minutes prior to incision? (Please mark all that apply):

- Availability of supplies/equipment for infusion

- Lack of an appropriate order

- Lack of IV access

- Don't feel it is beneficial/necessary to give prior to entry to OR

- Availability of the medication from pharmacy

- Other

13. Comments

Fig. 1 Provider survey regarding preoperative antibiotic prophylaxis

agreed that vancomycin infusions are completed at the time of surgery. Common barriers to timely administration of vancomycin prior to incision included issues with the availability of the medication from the pharmacy, the availability of equipment required for infusion, incorrect medication ordering, the lack of intravenous (IV) access for the patient, and other issues with the preoperative nursing staff.

\section{Attending orthopaedic surgery providers}

A total of 28 attending orthopaedic surgeons were approached to complete the survey. Twenty-three (82\%) attending orthopaedic surgeons completed the survey. Ten (44\%) attending orthopaedic surgeons agreed that vancomycin and cefazolin are equally effective for antibiotic prophylaxis whereas $12(52 \%)$ disagreed, and 1 (4\%) was unsure (Fig. 3). Nine providers (39\%) preferred vancomycin as the antibiotic choice for patients with a penicillin allergy, $8(35 \%)$ preferred clindamycin, and $6(26 \%)$ disagreed with both practices. All 23 (100\%) of the attending orthopaedic surgeons surveyed agreed that vancomycin should be dose adjusted by weight and 21 (91\%) agreed that cefazolin should be weight adjusted. The attending orthopaedic surgeon data also indicated 

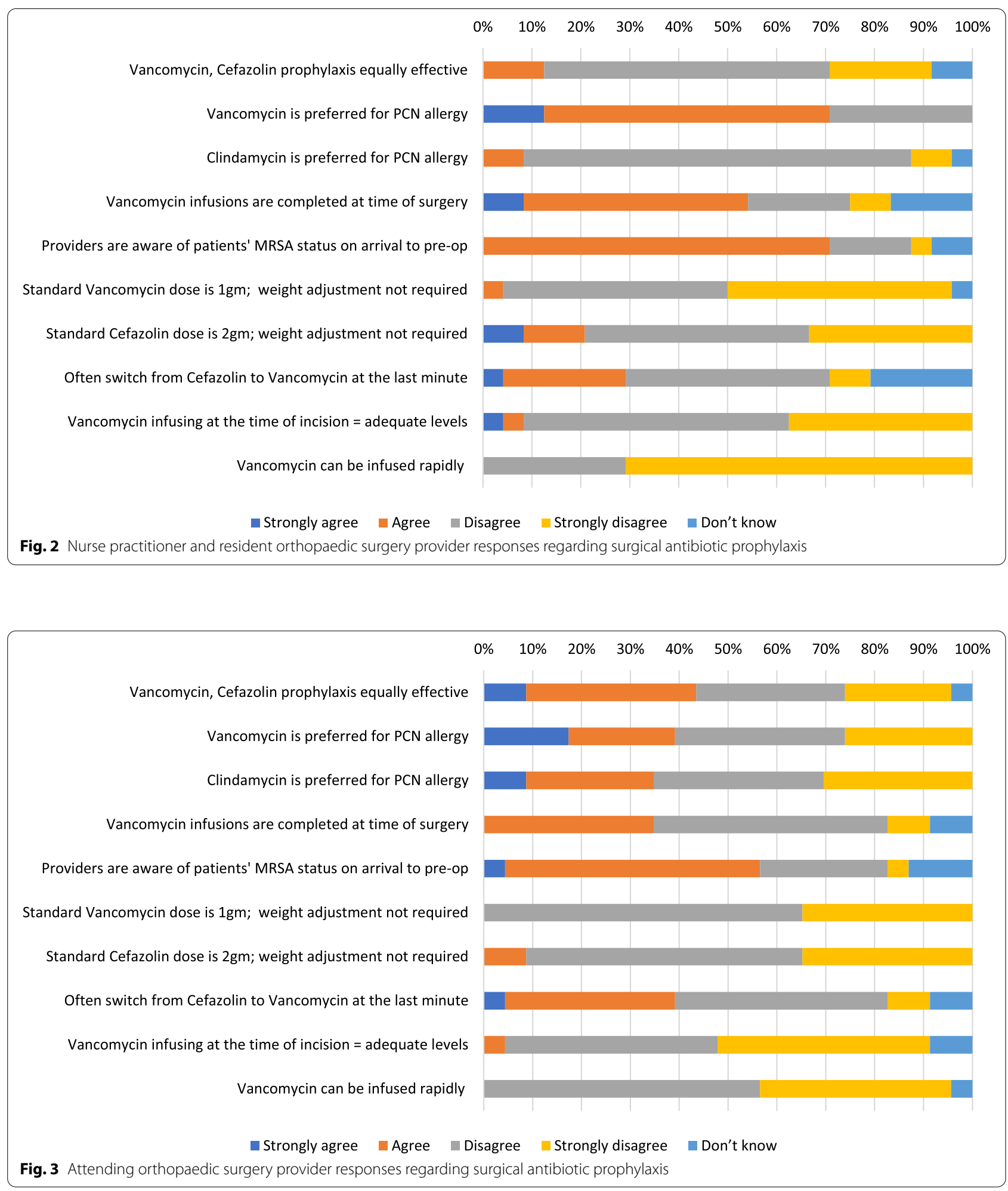

barriers to vancomycin's effectiveness as a suitable method for prophylaxis. Twenty-two (87\%) agreed that vancomycin infusion at the time of incision does not allow for adequate concentrations for appropriate antibiotic prophylaxis. In addition, 22 providers (96\%) recognized that vancomycin cannot be infused rapidly in order 
to maximize the proportion of dose infused prior to the time of incision. Furthermore, only 8 providers $(35 \%)$ agreed that vancomycin infusions are completed at time of surgery. Similar to the NP and resident data, additional reported barriers to timely administration of vancomycin prior to incision included issues with the preoperative nursing or anesthesia staff which delayed administration.

\section{Anesthesia providers (CRNA's, Residents, Attendings)}

Twelve CRNA's, 6 anesthesia residents, and 8 attending anesthesiologists completed the survey. Nine $(35 \%)$ anesthesia providers agreed that vancomycin and cefazolin are equally effective for antibiotic prophylaxis whereas 16 $(62 \%)$ disagreed, and 1 (4\%) was unsure (Fig. 4). Eleven $(42 \%)$ anesthesia providers preferred vancomycin as the antibiotic choice for patients with a penicillin allergy, 11 (42\%) preferred clindamycin, and 4 (15\%) disagreed with both practices. As for the question regarding standard versus weight adjusted dosing, 22 (85\%) anesthesia providers agreed that vancomycin should be dose adjusted by weight and similarly $23(88 \%)$ agreed that cefazolin should be weight adjusted. Similar to all of the other providers surveyed in this study, the anesthesia provider data also indicated barriers to vancomycin's effectiveness as a suitable method for prophylaxis. Nineteen $(73 \%)$ providers agreed that vancomycin infusion at the time of incision does not allow for adequate concentrations for appropriate antibiotic prophylaxis. In addition,
24 (92\%) providers recognized that vancomycin cannot be infused rapidly in order to maximize the proportion of dose infused prior to the time of incision. Furthermore, only $8(31 \%)$ providers agreed that vancomycin infusions are completed at time of surgery. Additional reported barriers to timely administration of vancomycin prior to incision included issues with the availability of vancomycin from the pharmacy, the availability of equipment required for infusion, lack of an appropriate order, lack of patient IV access, and issues with the preoperative nursing staff.

\section{Impact of service and role on survey responses}

For most survey questions, a large difference in responses between provider type (residents, attendings, NP's, or CRNA's) or service (anesthesia or orthopaedics) was not observed. However, there was significant disagreement regarding preferred agent for penicillin allergies, with residents more frequently indicating agreement with vancomycin (20 out of $28,71 \%$ agreement) compared to CRNA's/NP's (4 out of 14, 29\% agreement), and attendings (13 out of $31,42 \%$ agreement). There were also differences between anesthesia and orthopaedics regarding awareness of patients' MRSA status with 30 (64\%) orthopaedic providers in agreement that MRSA status is known and only $9(35 \%)$ anesthesia providers in agreement. Lastly, anesthesia was more likely to agree that frequent switches from cefazolin to vancomycin occur at

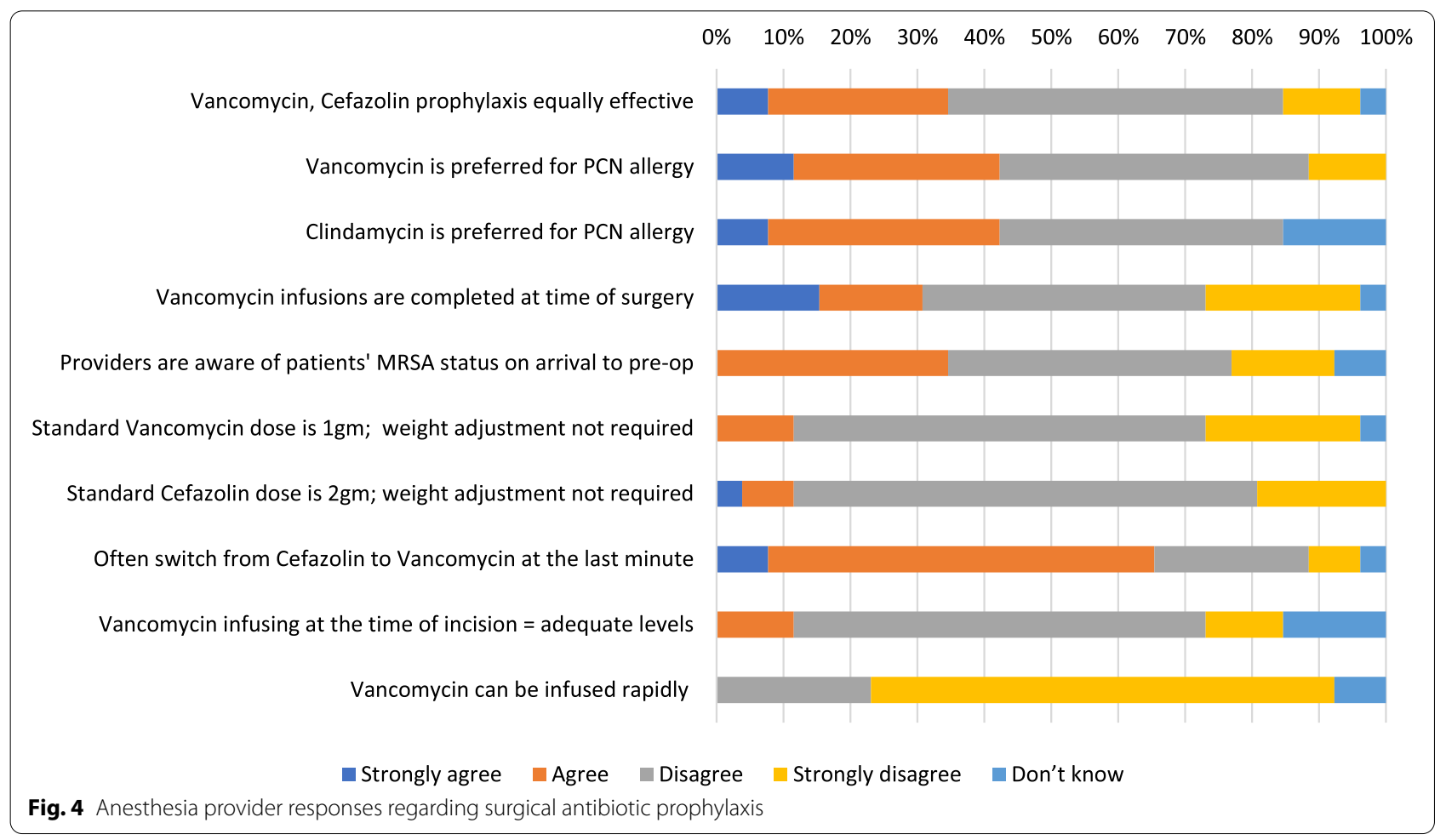


the last minute (17 out of $26(65 \%)$ in agreement versus 16 out of 47 (34\%) amongst orthopaedic providers).

\section{Discussion}

Despite well established guidelines and the known efficacy of antibiotic prophylaxis for reducing the risk of SSI, there continues to be wide variation amongst antibiotic prophylaxis practices. Therefore, we performed this study to assess the perspectives of providers at our institution regarding some of these practices including preoperative antibiotic choice, dosing, and timing. We determined that there is no clear consensus regarding the effectiveness of vancomycin and cefazolin for antibiotic prophylaxis since $30 \%$ of providers agreed and $64 \%$ disagreed that both antibiotics are equally effective. Similarly, there was also no consensus on the antibiotic choice for patients with a penicillin allergy since $51 \%$ of those surveyed agreed with vancomycin, $29 \%$ agreed with clindamycin, and the remaining $21 \%$ disagreed with both alternatives. In contrast, providers did generally agree with necessity of weight based dosing and timely infusion of vancomycin.

Overall, the results from this study indicate that there is no clear consensus amongst the providers at our institution when it comes to which antibiotic to administer for prophylaxis against SSI despite institutional guidelines developed by surgical service leadership. Several institutions from all over the world have determined that antibiotic prophylaxis is often inadequately administered. In a study from China that included 53 hospitals and a total of 14,525 procedures, $\mathrm{Ou}$ et al. determined that in only $9.4 \%$ of the procedures was antibiotic prophylaxis appropriate and correct in all steps, which included antibiotic choice, dose, dosing strategy, time of administration and duration of prophylaxis [10]. Similarly, Hawkins et al. performed a study involving 143 pediatric procedures and found that although $99 \%$ of the patients were correctly given or withheld prophylactic antibiotics, complete adherence to antibiotic guidelines was only present in $48 \%$ of cases [11]. In fact, weight-based dosing was present in only $77 \%$ of cases, timing of administration was correct in only $73 \%$ of cases, and only $7 \%$ of cases were appropriately re-dosed [11]. Similarly, in a study from France including 1,312 procedures, Muller et al. determined that non-compliance to the French national recommendations was evident in $44 \%$ of cases they studied [12]. In addition, specific to patients with betalactam allergies, Nguyen et al. demonstrated that among the cohort of patients they studied, only $37 \%$ of patients with labeled beta-lactam allergies received appropriate preoperative antibiotic prophylaxis compared to $76 \%$ appropriateness in patients without labeled allergies [13]. Therefore, although well-established antibiotic prophylaxis guidelines exist, great variability and poor compliance are major obstacles to adequate prophylactic antibiotic administration.

The explanations for our results are multifactorial. One reason for the lack of consensus in terms of appropriate antibiotic choice may be due to the fact that best practice guidelines are not widely displayed throughout preoperative and operative areas at our institution. Therefore, lack of awareness could be a potential contributor to our results. Another potential explanation is that since there is no formal education or training for both orthopaedic surgery and anesthesia team members regarding the topic of antibiotic prophylaxis, providers at our institution may not possess the most up-to-date knowledge in regards to this topic. Furthermore, as antibiotic resistance and drug allergies continue to increase in our communities, there is a need to continually educate health care providers on the most current literature available. Thus, an educational gap could be another contributing factor to our results. Lastly, there is also no antibiotic prophylaxis checklist at our institution to help standardize prophylaxis practices, which is often a key component of successful quality improvement initiatives [14].

In contrast to antibiotic choice, there was agreement at our institution that cefazolin and vancomycin dose should be weight adjusted. This consensus is most likely explained by the fact that the electronic medical record (EMR) at our institution prompts physicians to use weight-based dosing when ordering prophylactic antibiotics. We also determined that providers agreed that vancomycin infusion at the time of incision at our institution is often not adequate for antibiotic prophylaxis. This has severe implications because it is well documented that patients with inadequate vancomycin infusion have a significantly higher risk of SSI compared to patients where infusion is complete prior to incision. In a study by Cotogni et al. involving 741 cardiac surgery patients, patients where vancomycin infusion was violated (i.e. surgical skin incision was performed before the end of vancomycin infusion) had greater than five times increased odds of SSI compared to patients where vancomycin infusion was completed prior to incision [15]. Through our survey, we learned that this finding was most likely due to many factors such as problems with antibiotic availability from the pharmacy, missing infusion equipment in the preoperative areas, problems with the preoperative nursing staff, no EMR order, or a lack of patient IV access which delayed the start of antibiotic infusion.

Based on the results from this study, we have determined that there may be many potential areas for improvement at our institution when it comes to antibiotic prophylaxis. However, results of quality 
improvement programs to improve antibiotic prophylaxis have been mixed. In a study from the University of Texas at Houston, Putnam et al. implemented three cycles of interventions from 2011 to 2014 to improve antibiotic prophylaxis [16]. A few of their interventions included modifying their pre-incision checklist to include all four elements of antibiotic administration (i.e. type, dose, timing, redosing), assigning the anesthesia team the role of antibiotic administration, and distributing and displaying prophylaxis guidelines [16]. After the interventions, the researchers found that although redosing compliance significantly improved, overall adherence and adherence to the correct dose and timing was unchanged. Furthermore, antibiotic type errors significantly increased after the interventions [16]. Similarly, in a study from Australia, Knox and Edye compared preintervention antibiotic prophylaxis practices to compliance after implementation of an interventional program that included displaying prophylaxis guidelines in surgical areas and advertising appropriate prophylaxis practices throughout their institution [17]. After the intervention, the researchers determined that overall adherence was unchanged with adherence at $18 \%$ preintervention and $15 \%$ postintervention [17]. In a study from Canada by So et al., the researchers also compared preintervention antibiotic prophylaxis compliance to postintervention compliance [18]. Their interventions included posting antibiotic protocols in the operating room (OR), having only recommended antibiotics readily available in the $\mathrm{OR}$, educating resident physicians during orientation, including prophylactic antibiotics at time out, and both computerized alerts and emails to physicians when protocols were not followed [18]. In contrast to the studies mentioned above, the researchers found that within the field of orthopaedics, complete compliance to established guidelines drastically increased from $4.5 \%$ preintervention to $54 \%$ postintervention [18]. Furthermore, the greatest improvement was in regards to the duration of antibiotics, where compliance improved from $9.5 \%$ preintervention to $75 \%$ postintervention [18]. Similarly, in a study from Egypt, Saied et al. developed and taught a two-day curriculum designed to educate anesthesiologists and surgeons at five institutions about proper antibiotic prophylaxis practices, specifically focusing on the time and duration of antibiotic administration [19]. The researchers determined that compared to preintervention antibiotic prophylaxis practices, the optimal timing of the first dose significantly improved in three of the five institutions and the optimal duration of prophylaxis improved by $25 \%$ in all five institutions, postintervention [19]. Therefore, based on the studies mentioned above, implementation of a multifactorial quality improvement strategy that includes an educational component may be beneficial to improve antibiotic prophylaxis adherence.

Our study has several important limitations. First, the study is purely qualitative since we gathered provider data with the use of a questionnaire. Second, the purpose of this study was to only assess the antibiotic prophylaxis perspectives at our institution; we did not perform a retrospective analysis to determine the actual adherence to antibiotic prophylaxis guidelines at our institution. Therefore, although we found no clear consensus amongst our providers when it comes to which antibiotic to administer for prophylaxis against SSI, we cannot determine if this finding directly translates to poor adherence to antibiotic guidelines at our institution. Third, our study is subject to selection bias since our sample size of 73 providers is small and we only surveyed providers involved with the care of orthopedic patients. Furthermore, specific to anesthesia providers, although department meetings are mandatory, not all anesthesia providers were present and thus we were unable to approach all anesthesia team members at VCU Health to complete surveys. Lastly, it is important to mention that these results are based on responses from providers only at one institution.

\section{Conclusions}

Our survey indicated that there is no clear consensus amongst providers for which antibiotic to administer for prophylaxis against SSI despite existing internally developed and surgery-type specific guidelines. There is also great discrepancy between orthopaedic surgery and anesthesia providers in regards to appropriate antibiotic choice for patients with reported penicillin allergies. Therefore, based on our results, institutions should implement evidence-based protocols for preoperative antibiotic prophylaxis and continue to prospectively monitor compliance in order to identify any inconsistencies that could result in inappropriate antibiotic prophylaxis for patients.

\section{Abbreviations}

SSI: Surgical site infections; MSSA: Methicillin-sensitive Staphylococcus aureus; MRSA: Methicillin-resistant Staphylococcus aureus; CoNS: Coagulase-negative Staphylococci; VCU: Virginia Commonwealth University; IRB: Institution Review Board; NP's: Nurse practitioners; CRNA's: Certified registered nurse anesthetists; IV: Intravenous; EMR: Electronic medical record; OR: Operating room.

\section{Acknowledgements}

Not applicable.

\section{Authors' contributions}

GJG conceived of the presented research idea. EZ created the questionnaire used in the study and also performed both the data collection and analysis. $N A, E Z, M D$, and GMB prepared the manuscript draft with important intellectual input from SLK and GJG. All authors participated in critical revision of 
the manuscript draft. All authors have approved of the final manuscript to be published and the entirety of the submission.

\section{Funding}

There were no sources of funding for this research study.

\section{Availability of data and materials}

The data sets used and analyzed during the current study are available from the corresponding author on reasonable request.

\section{Declarations}

\section{Ethics approval and consent to participate}

The present research was submitted to the Institutional Review Board (IRB) at Virginia Commonwealth University prior to performing the study and was determined to be exempt.

\section{Consent for publication}

Not applicable.

\section{Competing interests}

The authors declare that they have no competing interests in relation to this work.

\section{Author details}

${ }^{1}$ Department of Orthopaedic Surgery, Virginia Commonwealth University School of Medicine, 1200 Broad Street, 9th Floor, VA 23298 Richmond, USA ${ }^{2}$ Department of Orthopaedic Surgery, Virginia Commonwealth University Health, VA 23298 Richmond, USA. ${ }^{3}$ Department of Infectious Disease, Virginia Commonwealth University Health, VA 23298 Richmond, USA.

Received: 29 April 2021 Accepted: 21 September 2021

Published online: 27 October 2021

\section{References}

1. Edwards JR, Peterson KD, Mu Y, Banerjee S, Allen-Bridson K, Morrell G, Dudeck MA, Pollock DA, Horan TC. National Healthcare Safety Network (NHSN) report: data summary for 2006 through 2008, issued December 2009. Am J Infect Control. 2009;37(10):783-805. https://doi.org/10.1016/j. ajic.2009.10.001. PMID: 20004811.

2. Southwell-Keely JP, Russo RR, March L, Cumming R, Cameron I, Brnabic AJ. Antibiotic prophylaxis in hip fracture surgery: a metaanalysis. Clin Orthop Relat Res. 2004;(419):179-84. https://doi.org/10.1097/00003086200402000-00029.

3. Ban KA, Minei JP, Laronga C, Harbrecht BG, Jensen EH, Fry DE, Itani KM, Dellinger EP, Ko CY, Duane TM. American College of Surgeons and Surgical Infection Society: Surgical Site Infection Guidelines, 2016 update. J Am Coll Surg. 2017;224(1):59-74. https://doi.org/10.1016/j.jamcollsurg.2016. 10.029 Epub 2016 Nov 30. PMID: 27915053.

4. Badia JM, Casey AL, Petrosillo N, Hudson PM, Mitchell SA, Crosby C. Impact of surgical site infection on healthcare costs and patient outcomes: a systematic review in six European countries. J Hosp Infect. 2017;96(1):1-15. https://doi.org/10.1016/j.jhin.2017.03.004 Epub 2017 Mar 8. PMID: 28410761.

5. Copanitsanou P. Recognising and preventing surgical site infection after orthopaedic surgery. Int J Orthop Trauma Nurs. 2020;37:100751. https:// doi.org/10.1016/j.ijotn.2019.100751 Epub 2019 Dec 18. PMID: 31954634.

6. AlBuhairan B, Hind D, Hutchinson A. Antibiotic prophylaxis for wound infections in total joint arthroplasty: a systematic review. J Bone Joint Surg Br. 2008:90(7):915-9. https://doi.org/10.1302/0301-620X.90B7.20498

7. Bratzler DW, Houck PM, Richards C, Steele L, Dellinger EP, Fry DE, Wright C, Ma A, Carr K, Red L. Use of antimicrobial prophylaxis for major surgery: baseline results from the National Surgical Infection Prevention Project.
Arch Surg. 2005;140(2):174-82. https://doi.org/10.1001/archsurg.140.2. 174. PMID: 15724000 .

8. Bratzler DW, Dellinger EP, Olsen KM, Perl TM, Auwaerter PG, Bolon MK, Fish DN, Napolitano LM, Sawyer RG, Slain D, Steinberg JP, Weinstein RA. American Society of Health-System Pharmacists; Infectious Disease Society of America; Surgical Infection Society; Society for Healthcare Epidemiology of America. Clinical practice guidelines for antimicrobial prophylaxis in surgery. Am J Health Syst Pharm. 2013;70(3):195-283. https://doi.org/10. 2146/ajhp120568. PMID: 23327981.

9. Mankowski P, Cherukupalli A, Slater K, Carr N. Antibiotic prophylaxis in plastic surgery correlation between practice and evidence. Plast Surg. 2021;29(2):132-8. https://doi.org/10.1177/2292550321997005 Epub 2021 Mar 2. PMID: 34026678; PMCID: PMC8120557.

10. Ou Y, Jing BQ, Guo FF, Zhao L, Xie Q, Fang YL, Cui J, Xiao W, Wu DW, Zhou W. Audits of the quality of perioperative antibiotic prophylaxis in Shandong Province, China, 2006 to 2011. Am J Infect Control. 2014;42(5):51620. https://doi.org/10.1016/j.ajic.2014.01.001 Epub 2014 Mar 20. PMID: 24656785.

11. Hawkins RB, Levy SM, Senter CE, Zhao JY, Doody K, Kao LS, Lally KP, Tsao K. Beyond surgical care improvement program compliance: antibiotic prophylaxis implementation gaps. Am J Surg. 2013;206(4):451-6. https://doi.org/10.1016/j.amjsurg.2013.02.009 Epub 2013 Jun 27. PMID: 23809676.

12. Muller A, Leroy J, Hénon T, Patry I, Samain E, Chirouze C, Bertrand X. Surgical antibiotic prophylaxis compliance in a university hospital. Anaesth Crit Care Pain Med. 2015;34:289-94. https://doi.org/10.1016/j.accpm.2015.04. 004.

13. Nguyen CT, Petrucci K, Daily E, Brown AM, Pettit NN, Pisano J. Investigating the impact of a $\beta$-lactam allergy label on preoperative antibiotic prophylaxis administration. Infect Control Hosp Epidemiol. 2021;42(6):710-4. https://doi.org/10.1017/ice.2020.1271 Epub 2020 Nov 17. PMID: 33198838.

14. Guerrero MA, Anderson B, Carr G, Snyder KL, Boyle P, Ugwu SA, Davis M, Bohnenkamp SK, Nfonsam V, Riall TS. Adherence to a standardized infection reduction bundle decreases surgical site infections after colon surgery: a retrospective cohort study on 526 patients. Patient Saf Surg. 2021;15(1):15. https://doi.org/10.1186/s13037-021-00285-7 PMID: 33832533; PMCID: PMC8034065.

15. Cotogni P, Barbero C, Passera R, Fossati L, Olivero G, Rinaldi M. Violation of prophylactic vancomycin administration timing is a potential risk factor for rate of surgical site infections in cardiac surgery patients: a prospective cohort study. BMC Cardiovasc Disord. 2017;17(1):73.

16. Putnam LR, Chang CM, Rogers NB, Podolnick JM, Sakhuja S, Matusczcak M, Austin MT, Kao LS, Lally KP, Tsao K. Adherence to surgical antibiotic prophylaxis remains a challenge despite multifaceted interventions. Surgery. 2015;158(2):413-9. https://doi.org/10.1016/j.surg.2015.04.013 Epub 2015 Jun 6. PMID: 26054317.

17. Knox MC, Edye M. Educational antimicrobial stewardship intervention ineffective in changing surgical prophylactic antibiotic prescribing. Surg Infect. 2016;17(2):224-8. https://doi.org/10.1089/sur.2015.194.

18. So JP, Aleem IS, Tsang DS, Matlow AG, Wright JG. SickKids Surgical Site Infection Task Force. Increasing compliance with an antibiotic prophylaxis guideline to prevent pediatric surgical site infection: before and after Study. Ann Surg. 2015;262(2):403-8. https://doi.org/10.1097/SLA.00000 00000000934.

19. Saied T, Hafez SF, Kandeel A, El-kholy A, Ismail G, Aboushady M, Attia E, Hassaan A, Abdel-Atty O, Elfekky E, Girgis SA, Ismail A, Abdou E, Okasha O, Talaat M. Antimicrobial stewardship to optimize the use of antimicrobials for surgical prophylaxis in Egypt: a multicenter pilot intervention study. Am J Infect Control. 2015;43(11):e67-71. https://doi.org/10.1016/j.ajic. 2015.07.004 Epub 2015 Aug 25. PMID: 26315059.

\section{Publisher's Note}

Springer Nature remains neutral with regard to jurisdictional claims in published maps and institutional affiliations. 